\title{
Preventive and exploratory: two workplace problem-solving cultures
}

\author{
Jacob Poirier, Vina Macias, Benjamin M. Zwickl, and Susan Rothwell \\ School of Physics and Astronomy, Rochester Institute of Technology, 84 Lomb Memorial Drive, Rochester, NY, 14623
}

The EMPOWER project conducted 186 interviews with employers, faculty, and students across nursing, manufacturing, computing, energy, and physics. The interviews discussed the problems faced across different workplaces and how each tries to resolve them. Two different cultural approaches to problem-solving were found. One culture values proactively identifying anomalies deviating from the norm while another values exploring creative solutions. The presence of risk and/or expense of an operation leads preventive problemsolvers to construct hypothetical situations and attempt to solve problems before they happen. An exploratory culture fosters creative ideas and experimentation in low-risk environments where failure is an acceptable part of the problem-solving process. The two approaches have parallels to physics education. The problem-prevention culture, which requires analyzing and addressing hypothetical anomalies, is similar to theoretical practices in physics. The exploratory culture of encouraging creative solutions is similar to experimental or design work within physics. (Supported by NSF DGE-1561493)

2019 PERC Proceedings edited by Cao, Wolf, and Bennett; Peer-reviewed, doi.org/10.1119/perc.2019.pr.Poirier Published by the American Association of Physics Teachers under a Creative Commons Attribution 4.0 license. Further distribution must maintain attribution to the article's authors, cover page, and DOI. 


\section{INTRODUCTION}

Organizations have specific cultures, which affects their operation. Maanen and Shein describe organizational culture as consisting of "long-standing rules of thumb", a "special language and ideology that help edit a member's everyday experience", "shared standards of relevance as to the critical aspects of the work", and some residual category of 'rather plain 'horse sense' regarding what is appropriate and 'smart' behavior within the organization and what is not" [1]. One example of an organizational culture would be safety culture, which is "an organization's values and behaviors - molded by its leaders and internalized by its members - that serve to make [...] safety the overriding priority" [2]. Within this study we examined how workplace problemsolving is shaped by the values, norms, and shared experiences within organizations. Two predominant organizational cultures emerged from examining how different disciplines across STEM solve problems. A preventive problem-solving culture emerged in disciplines such as energy and nursing which tried to maintain a desired state. Explorative problemsolving culture emerged primarily in the fields of computing and advanced manufacturing, which both valued generating multiple solutions, testing, failing, and iterating.

\section{METHODS}

In four high STEM metropolitan areas across the US, 186 interviews were conducted. Faculty at two- and four-year institutions (92 interviews) and employers (25 interviews) in industry were interviewed individually and students were interviewed in focus groups (69 focus groups with 239 students). The disciplines studied in this project were nursing, manufacturing, computing, energy, and physics. All interviews were semi-structured, consisting of a free list for competencies required in their field, defining teamwork, communication, problem solving, and self-directed learning, and where they learned or taught the four competencies. Initially, an a priori coding approach captured broad aspects of problems-solving. The code "definition of problem" (how an interviewee describes a problem they face) yielded 1010 instances, "strategies to solve" (a method or characteristic of a problem solver) yielded 1257 instances, and "teaching" (how problem-solving is taught) yielded 1390 instances. Two cultural approaches to solving problems emerged from the a priori coding, preventive and explorative cultures. A series of text searches for the phrases "prevent" (516 references) "fix" (136 references), and "troubleshoot" (123 references), was then conducted across all interviews. The results from the a priori coding and text searches were analyzed through a process of Initial Coding (i.e., open coding) [3]. Out of 1870 open codes related to problem-solving, 452 were selected for their relevance to preventative (i.e., solving problems before they happen) or explorative (i.e, designing, creating, or iterating upon solutions) cultures. General discussions of problem-solving (e.g., criti- cal thinking) and technical skills not related to preventive or exploratory problem-solving (e.g., math) were excluded. The 452 remaining Initial Codes were imported into visualization software, sorted into a dichotomy of prevention and exploration and then thematically grouped.

\section{PREVENTIVE CULTURE}

A preventive problem-solving culture has a desired state they wish to preserve and will focus its efforts on maintaining that norm, as extreme variance from the norm may lead to catastrophe. A preventive culture can incorporate aspects of safety culture. Two fields within our study that commonly employed a preventive culture were petroleum engineering and nursing.

\section{A. Complexity}

A preventive culture combines constant vigilance with large scale data analysis and ongoing monitoring to attempt to maintain the system. An example from the petrochemical industry was that a plant was "running an incredibly complex amalgamation of machines and instrumentation" where a board operator was "problem-solving at the level of trying to allow the problem not to manifest before we solve it" (petrochemical engineering instructor). As soon as an anomalous change deviates from the norm, the operator's job was to correct it. When this was successful, no problem ever actually occurred.

A difficulty found in petrochemical plants was their lack of downtime. The plants ran 24 hours a day with shifts lasting 12 hours. The continuous operation forced maintenance and efficiency. There was a cycle of inspection and repair that kept the plant operating because plant operators had learned "if you can't be safe, it's going to impact the amount of time the unit runs, which impacts the profitability" (petrochemical engineering instructor). This couples two of the main drivers of the preventive culture in the petrochemical processing industry: safety followed by expenses. It was necessary for petrochemical operations to always maintain everyone's safety, but it needed to efficiently generate useful product as well. This desire to constantly produce chemicals led to continuous operation, which motivated a preventive approach to avoid any problems that might occur.

\section{B. Uncertainty}

Some interviewees described the uncertainty of dealing with large complicated systems. In petroleum engineering, there could be large variations in the specifics between any two wells (e.g., oil wells). Each well required problemsolving for their individual requirements. 
"not every single one of those is going to be the exact same. [...] One might run for three years; one might run three days" (petrochemical engineering student)

The uniqueness of wells means each one needed its own solution to its creation and production. This was similar to a difficulty faced by geological engineers in that there were no defined formulas for them to use to solve problems. They had to "use all these ambiguous things and combine all that together" (geological engineering student), looking across all of their resources and performing careful, individual analysis of their specific problem.

Nursing also involved uncertainty, particularly when first encountering a patient.

"The patient comes to us with a problem. They wouldn't need us if they didn't have a problem. And they can't say, "Hey, I've got blood filling up in my pleural cavity and now I can't breathe." They just come in there and they can't breathe and they're not talking to you because they're focusing on that they can't breathe." (nursing student)

Nurses had to figure out what symptoms the patient has through verbal and nonverbal communication, building a medical background for a patient.

\section{Safety}

A preventive culture often embraced a safety culture. An instructor from petroleum engineering stated:

"Fundamentally, people from the outside would view our job as operating these units to modify a chemical precisely into something a customer would buy. [...] I guess fundamentally, that's our job, but at the same time, we're responsible for the safety of everyone entering that unit to perform all the ancillary work that has to be done to keep that unit running" (petrochemical engineering instructor)

Deliberate action was required to maintain the safety of the personnel. Proper reporting of everything that went on was also necessary because what appeared to be a small discrepancy could spread throughout the plant. A foundation of safety culture is continuously improving from any mistakes, especially the small ones that could occur day-to-day and establishing measures to stop them.

Nursing also embraced safety culture due to the potential danger inherent in helping the sick. A common discussion across all interviewees was the importance of remembering lives are at stake. The threat of loss of life forced nurses to come together in a crisis. "It's not just one nurse trying to save this person's life, it's a whole team of people who are called in" (nursing student). This follows one of the foundational tenants of safety culture, every member of the culture must be committed to safety and be ready to act during a crisis.

\section{Preventive Collaboration}

A board operator in a chemical plant was constantly monitoring the state of the plant. When a deviation away from normal operating conditions occurs, they started to ask themselves a series of questions to understand the situation.

"Say, if you're looking at your board, and all of a sudden, your pump starts reading high, okay, it's running hot. [...] am I getting flow to it, when was the last time this pump was maintained? Or big things of pressure? Okay, if somebody looks at a tank and they just see a high-pressure reading, well, if that's as far as their mind can see, is a high-pressure reading, then they're going to get somebody hurt, because they're not going to be able to make adjustments." (petrochemical instructor)

Once they had an understanding of the situation, they could start to implement a solution. Because the board operator observes remotely, their relationship with technicians was very important. Technicians were inside the plant and acted as the operator's eyes and hands, finding, fixing, and confirming any discrepancies the operator identified. There would often be the discussion of trust within these teams.

"All you can do is just talk over a radio and they have to trust you enough that you know what you're talking about and they can just do what you say" (petrochemical engineering student)

One structure put in place to help these teams was repeatback communication. The operator would request the technician to preform a procedure such as opening a valve and the technician would repeat back the request. The operator would then confirm that it was correctly interpreted, the technician would open the valve and state that they did. This formalized communication was used to prevent miscommunication which could lead to equipment failure if an incorrect procedure was carried out, presenting a danger to both the personnel and the plant.

Nurses had to act as the liaison between their patients and the rest of the medical community that supported them. They had to maintain the records of the patient that would be dispersed to any doctors, surgeons, or specialists that the patient might see, as well as across different shifts. In this setting, it was desirable to record "short, fragmented sentences" that accurately captured the patient's condition. For example, "Patient laying in bed. Respiration is even, unlabored. Eyes closed" (nursing student). The short descriptions were used to provide easy-to-read written information. Multiple members 
of the medical community interacted with a patient throughout their time at the hospital, possibly across multiple shifts. Miscommunication or missing information puts the patient at risk.

\section{EXPLORATORY CULTURE}

An exploratory problem-solving culture encouraged multiple creative solutions and an iterative cycle of testing and synthesis, often without worrying about the possibility of failure. An exploratory culture had lower stakes in terms of the risks or costs associated with problem-solving. Computing and manufacturing were two fields in this study which were typically situated in an exploratory culture.

\section{A. Acceptability and Usefulness of Failure}

An exploratory culture had a mindset of build, then fix, then break, then rebuild, and iterate. This acceptability of failure was what powers the plethora of solutions that could be tested and refined. This mindset of encouraging failure can only exist in a culture that exhibited relatively low risk and low cost in the problems they face. This let problem solvers "want to fail" so they could find the best solution that works (manufacturing student). In order to motivate exploratory problem-solving an organization had to "have the right cultural conditions where it's okay to highlight imperfection" with a "glass half full notion" towards problems as opportunities for improvement (computer science instructor).

Sometimes an exploratory culture could occur because the problems are just difficult. The division of time during the creation of a computer program was described as " $10 \%$ of your time writing that program and $90 \%$ bug fixing" (computer science student). A student described their past experiences with programming as "I've been programming for five years and never once have I written a program that has worked on the first try. It just hasn't happened. So just learn to expect those errors and being able to fix bugs." (computer science student). These two quotes encompass the ideas of an exploratory culture, combining the iterative process of refining a product with the ability to fail to push improvements.

Pushing solutions until they break was another aspect of exploratory problem-solving.

"Engineers have to be skeptical [...] We're being taught that as engineers, failure is our job. Make things break." (manufacturing student)

Once there was the ability to fail, it could be leveraged to make better and better solutions. Intentionally breaking the product to find out when it fails to iterate on its design could only be afforded when there was both low risk and low cost associated with the break.

The cost of materials had a significant impact on the problem-solving process an industry would carry out. For mass produced circuit boards, companies would "throw the board out and just put a whole new board in" if there was a defect present, simply because it was cheaper to produce a new one than to spend the time having a person troubleshoot the faults (manufacturing instructor). This provided an inexpensive, fast, and easy solution to their problem only available when failure was acceptable.

\section{B. Seeking multiple solutions}

An exploratory culture valued generating ideas. In order to get the best solution, many alternatives needed to be considered.

"It takes 10 ideas to come up with one good one. It takes 100 ideas to come up with a great one. It takes 1,000 ideas to come up with a groundbreaking one." (optical engineer)

An exploratory culture tackled open questions in low-risk environments, which let the problem solvers play with their solutions. Since the environment allows for the testing of multiple solutions, the best could be discovered and developed into the strongest implementations.

Information Technology (IT) displayed a facet of an exploratory culture with interactions between a client and technical support staff. A client using the program could encounter a problem and seek help from an IT professional. The client could be using the product in unconventional ways or pushing the boundaries of its expected use. That use outside of the programs anticipated application could lead to the development of new ideas to satisfy the client.

\section{Optimization on the fly}

Within advanced manufacturing, some problems could be solved on-the-fly. "If your speeds and feeds are wrong, you're going to have chatter" (machining student). To correct for this chatter, the machinist had to make adjustments in the process of making the part. They could override the set parameters for the part based on what they saw or heard, tweaking the setup "on the fly". This allowed a skilled machinist to start tackling a problem without a fully formed solution, letting them rely on their personal expertise to guide their process. This could be the most effective way to produce a part because of the difficulties creating an automated or entirely thought out process for manufacturing the part.

\section{COMPARISONS}

\section{A. Risk and Cost}

The largest divide between the two cultures was their ability to handle risks or costs. A preventive culture worked under 
conditions where sloppy work increased the chance for injury, loss of life, or large financial losses, and failure is avoided. An exploratory culture often lacked these risky conditions. For instance, a computer program can be copied, tweaked, modified, and rerun without any considerable loss, whereas a petrochemical plant cannot be easily modified merely to experiment with a new procedure that might not work. A nurse should avoid a trial and error approach to helping a patient, whereas an optical engineer can take the time to attempt many trials, adjusting and realigning the components as they go.

\section{B. Troubleshooting}

Preventive and exploratory cultures both used the term troubleshooting to describe some aspects of problem-solving, but each had a unique approach. A preventive culture had a highly structured system for troubleshooting, often necessitated because of the risk faced if the troubleshooting fails. If the temperature spikes, a petrochemical operator would ask: did a pipe burst, what's going in and out of the piece of equipment, and did the pressure change, all without neglecting any other information that they were observing. Within an exploratory culture it was permissible for an engineer to experiment with the problem, even break their product in the process of finding out what is wrong, which a preventive culture must avoid at all costs.

\section{Users and Communication}

Preventive and exploratory cultures tend to have very different populations of users. In a preventive culture with a high-stakes, high-tech environment where complex production technology is the area of risk (petrochemical plant), users are the workers in the high-stakes environment. The user focus is on protecting individuals involved in high stakes production of an end product, and if a catastrophe could impact nearby areas, on protecting public health and safety. In an exploratory culture such as IT, users are the clients using the end products (software), and the user focus is on meeting client needs. This difference in user population was reflected in the ways preventive and exploratory cultures used communication. A preventive culture incorporated communication into its strategies to avoid problems, as a structured tool to convey information that could be dangerous if left out or miscommunicated. In addition to task-specific communication such as repeat-back, communication in a preventive culture should include ensuring that all users (plant personnel) are made aware of and educated on problems or changes that could impact them. An exploratory culture used communication for evaluation during product design (critiquing ideas) or when assisting a client after a product failure. Evaluation may involve extended dialogue with a user unfamiliar with the product. A client (software user) might not be fully aware of the problem experienced, and the IT professional needs to figure out the clientâs difficulty and communicate a solution in a way the client can understand. âYou [...] weed through what theyâre telling youâ (computing student) The IT professional had to process what they were told. This was an entirely different skill from operator-technician communication with a structure specifically built to avoid interpretation.

\section{CONCLUSIONS}

Two predominant problem-solving cultures were identified in this analysis of problem-solving across multiple STEM fields. However, there were a few limitations to the identification of these two cultures. There was not always a strict dichotomy between preventive and exploratory cultures. Each may use certain strategies or methods taken from the other. Nursing and manufacturing used strategies from both of the cultures. The interaction between a nurse and a patient has some parallels to the kind of problem-solving used by IT professionals when interacting with clients. Additionally, in manufacturing, once a product moved from development into large-scale production there was a shift in perspective toward being error-free to maximize efficiency. Even with the overlaps, the central focus of nursing was still on keeping the patient healthy and the focus of manufacturing was coming up with the best solution, placing them into the preventive and exploratory cultures.

These cultures may have relevance to undergraduate education. Key features of a preventive culture are complexity, a focus on safety, and maintaining normal operation while minimizing risk. An undergraduate research laboratory would be a good place to introduce physics students to this culture. Research labs could provide the technological depth and interconnections that could be found in industry. It also provides an environment where students can be exposed to more comprehensive safety procedures, using expensive equipment, and obeying important procedures.

Key features of the explorative culture include the acceptability of failure and encouraging many possible solutions. Electronics or computational labs are two spaces where an exploratory approach could be applied within a physics education. Both topics provide students a space where they can come up with their own solutions and evaluate them, and recognize that failure can be an acceptable and beneficial part of the problem-solving process.

\section{ACKNOWLEDGMENTS}

The study design and data collection was done in conjunction with Matt Hora, Ross Benbow, Bailey Smolarek and other collaborators at the Wisconsin Center for Education Research. 
[1] J. V. Maanenand E. H. Schein, Toward a theory of organizational socialization (1979).

[2] Principles for a Strong Nuclear Safety Culture, Tech. Rep. (Institute of Nuclear Power Operations, 2004).
[3] J. Saldana, The Coding Manual for Qualitative Researchers, second edition ed. (SAGE Publications Ltd, Los Angeles, 2012). 